\title{
Studies toward the synthesis of angularly-oxygenated angucyclines antibiotics
}

\author{
Nathalie Lebrasseur, $^{a}$ Gao-Jun Fan, ${ }^{b}$ and Stéphane Quideau* ${ }^{a, b}$ \\ ${ }^{a}$ Institut Européen de Chimie et Biologie, Pôle Chimie Organique et Bioorganique, 2 rue Robert \\ Escarpit, F-33607 Pessac Cedex, \\ ${ }^{b}$ Laboratoire de Chimie des Substances Végétales, Centre de Recherche en Chimie Moléculaire, \\ Université Bordeaux 1, 351 cours de la Libération, F-33405 Talence Cedex \\ E-mail: s.quideau@iecb-polytechnique.u-bordeaux.fr
}

Dedicated to Professor Karsten Krohn on the occasion of his $60^{\text {th }}$ birthday

(received 31 Mar 04; accepted 17 Aug 04; published on the web 23 Aug 04)

\begin{abstract}
The [bis(trifluoroacetoxy)]iodobenzene-mediated oxidative dearomatization of 2-alkoxyarenols, followed in situ by trapping of the resulting arenoxenium ions by soft external carbon-based nucleophiles, constitutes a rapid access to highly functionalized naphthoid cyclohexa-2,4dienones. These synthons can serve as valuable intermediates in the construction of the angularly-oxygenated benz[a]anthraquinone ABCD tetracyclic ring system of aquayamycin-like angucyclinones. This methodology so far has led to the elaboration of five-membered ring analogues of the ABC tricyclic unit of these natural products.
\end{abstract}

Keywords: Angucyclines, aquayamycin, dearomatization, cyclohexa-2,4-dienones, $\lambda^{3}$-iodane

\section{Introduction}

Angucyclines along with tetracyclines and anthracyclines are the third class of natural antibiotics featuring a carbotetracyclic skeleton. ${ }^{1,2}$ These natural products are isolated from the fermentation broth of Actinomycetes; they feature a benz $[a]$ anthraquinone framework with varying degree of insaturation and oxygenation, and display a broad spectrum of biological activities including antitumor, antifungal and antiviral properties. ${ }^{1,2}$ Their structural diversity has provided organic chemists with attractive targets for the development of various synthetic methodologies. Until now, most synthetic efforts have been directed toward angucyclines with an aromatic B ring. In most cases, the strategy used for constructing the benz $[a]$ anthracene framework is based on the Diels-Alder reaction, ${ }^{3-12}$ but other synthetic approaches also have been described. ${ }^{13-22}$ However, angucyclines bearing two hydroxy groups at the $\mathrm{AB}$ ring junction positions (Figure 1) hold a 
special status among these targets because (1) their total synthesis still constitutes a real challenge and (2) their biological activities ${ }^{23-29}$ are the most therapeutically significant ones e.g., exhibiting antitumor activity against adriamycin- and doxorubicin-resistant P388 leukemia cells. ${ }^{1,30}$ In this subclass, two groups are distinguished: angucyclines without and with a C-glycosidic moiety, exemplified by the angucyclinones SS-228Y (1) and aquayamycine (2), respectively. Inhibition of dopamine $\beta$-hydrolase has been reported for both $1(62.5 \%$ at $0.29 \mu \mathrm{M})^{31}$ and $2(50 \%$ at $0.40 \mu \mathrm{M}){ }^{23}$ Aquayamycin (2) is also known as an inhibitor of tyrosine hydroxylase $(50 \%$ at $0.37 \mu \mathrm{M}){ }^{24}$ Vineomycin $\mathrm{A}_{1}(3)^{32}$ exhibits antitumour activity against Sarcoma 180 solid tumour in mice, and SS-228Y (1) also was found to show cytotoxic activity in vivo. $^{31,33}$ Saquayamycin A (4) ${ }^{28}$ and ritzamycin A (5) display remarkable activities against L1210 and HT-29 tumor cells, even though certain saquayamycins have shown some levels of toxicity in vivo.

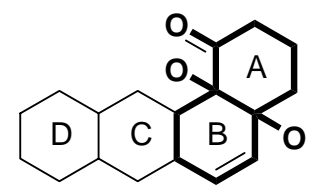

angucycline antibiotic skeleton with angular oxygen atoms

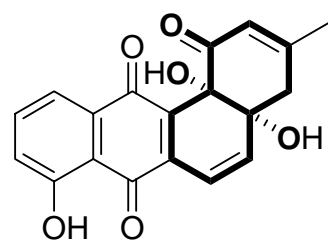

1: SS-22BY

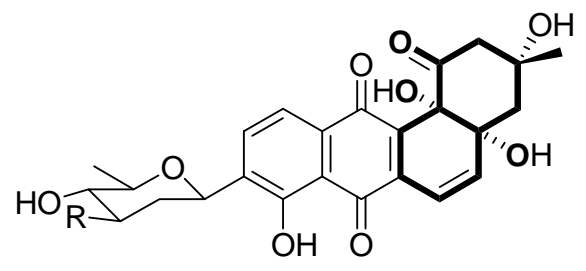

2: $\mathrm{R}=\mathrm{OH} ;$ aquayamycin

5: $\mathrm{R}=\mathrm{H}$; ritzamycin A

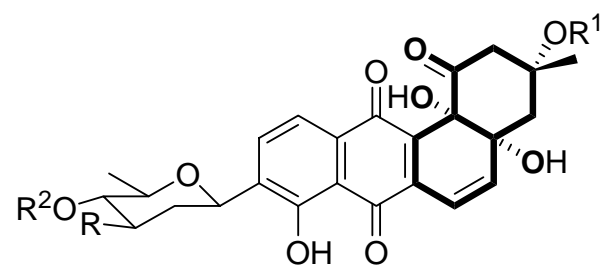

3: $R^{1}=A^{1}, R^{2}=H$; vineomycin $A_{1}$

4: $R^{1}=H, R^{2}=A^{2}$; saquayamycin $A$

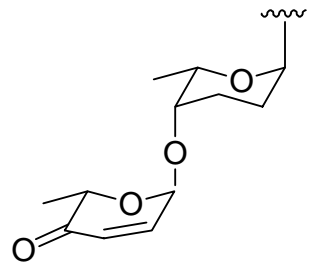

$A^{1}$

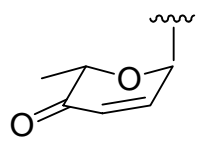

$A^{2}$

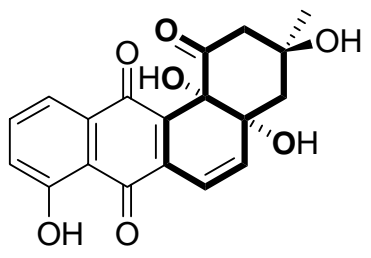

6: WP 3688-2

\section{Figure 1}

Only two total syntheses of such angucyclines have been reported: Krohn's synthesis of the racemic 8-deoxy analogue of WP 3688-2 (6) ${ }^{34}$ and the first total synthesis of aquayamycin (2), 
described by the Suzuki group. ${ }^{35-37}$ In both cases, the key A ring annulation step was a pinacoltype coupling. Our previous investigation ${ }^{38,39}$ on the formation of naphthoid cyclohexane-2,4dienones via $\lambda^{3}$-iodane-mediated oxidation of 2-alkoxynaphthols such as $\mathbf{9}$ followed in situ by regioselective attack of a carbon-based nucleophile such as 10, led us to design a new synthetic approach toward the benz[a]naphthalene ABC unit of angularly oxygenated angucyclines (Scheme 1). We report herein some preliminary results from this novel approach that led to the elaboration of five-membered ring-containing analogues of this angucyclinone unit.

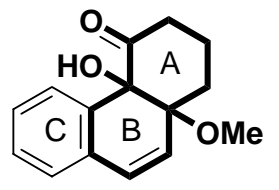

7<smiles>COC1(C/C(C)=C/C=O)C=Cc2ccccc2C1=O</smiles>

8<smiles>C=C(C)/C=C/OC(C)C</smiles>

10

\section{Scheme 1}

\section{Results and Discussion}

The regioselective formation of naphthoid cyclohexa-2,4-dienones by [bis(trifluoroacetoxy)]iodobenzene-mediated (BTI) oxidative dearomatization of 2-alkoxynaphthols in the presence of external carbon-based nucleophiles is a synthetically valuable process that we have recently introduced. ${ }^{38,39}$ The presence of a strong electron-releasing group, like an alkoxy group at the 2-position of the starting naphthol, is essential for regiochemical control of this reaction. In the context of the synthesis of aquayamycin (2) and its congeners, we used the silyl enol ether 10, which presents the advantage of furnishing in one step a four-carbon side-chain adequately functionalized for the A ring annulation. A mixture of 2-methoxynaphthol (9) and diene 10 was thus treated with BTI (1.8 equiv) in $\mathrm{CH}_{2} \mathrm{Cl}_{2}$ at $0{ }^{\circ} \mathrm{C}$ for two hours to furnish both the $\mathrm{C}-2$ (8) and the $\mathrm{C}-4$ (11) adducts in 36\% and 13\%, respectively (Scheme 2).

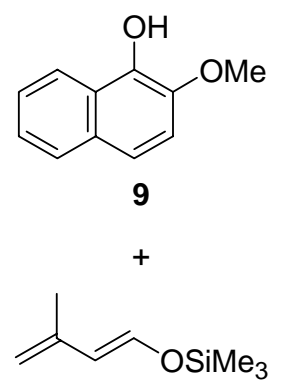

10
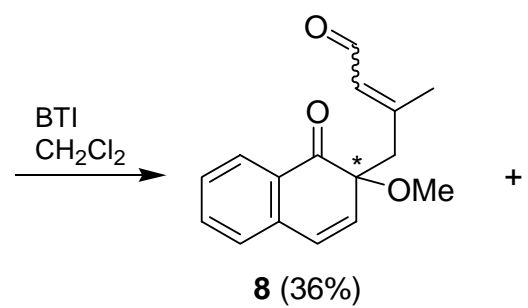

$E / Z(1.3: 1)$<smiles>COc1cc(C/C(C)=C/C=O)c2ccccc2c1O</smiles>

$11(13 \%)$

\section{Scheme 2}


The desired ortho-quinol ether 8 was obtained in $36 \%$ yield as a 1.3:1 mixture of $(E)$ - and (Z)-isomers as determined by NOE spectroscopy. We must emphasize the conciseness of this approach to highly functionalized ortho-quinol ethers that can then be used for annulation to the $\mathrm{ABC}$ ring system of angucyclinones. The first annulating strategy we considered was an intramolecular thiazolium ion-catalyzed aldehyde-ketone benzoin-type condensation (Scheme 3). ${ }^{40,41}$ No cyclization was observed under the conditions applied, the only product isolated after heating 8 at $80{ }^{\circ} \mathrm{C}$ in absolute ethanol and in the presence of thiazolium chloride $\mathbf{1 2}$ and triethylamine was the thermally-induced Cope rearrangement product 16 (90\%) (Scheme 3). The reaction performed at lower temperature only led to the recovery of starting material.
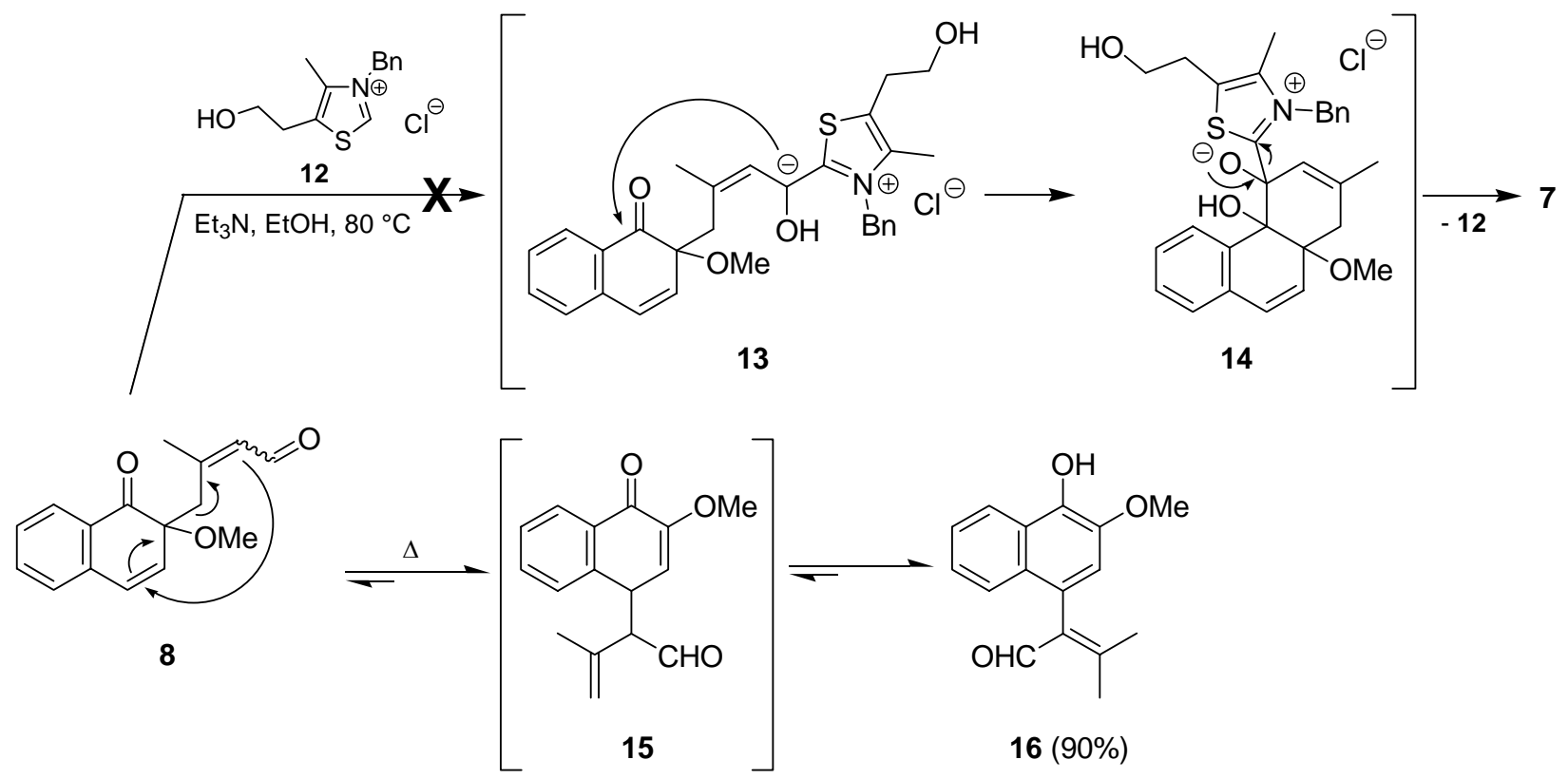

\section{Scheme 3}

The next strategy we examined was derived from Kraus' synthesis of an aquayamycin-type $\mathrm{ABC}$ ring system; ${ }^{22}$ the construction of the $\mathrm{A}$ ring was based on the conversion of a $\mathrm{B}$ ringtethered aldehyde into a cyclizing acyl carbanion equivalent. Thus, trimethylsilyl cyanide was added to $\mathbf{8}$ in the presence of catalytic amounts of potassium cyanide and 18-crown-6, thereby furnishing the protected cyanohydrin 17 in 37\% yield (Scheme 5). Treatment of 17 with LDA in THF at $-78{ }^{\circ} \mathrm{C}$ restored the aldehyde 8. This somewhat surprising recovery can be explained simply by deprotonation at one of the $\gamma$-allylic positions in place of the cyanohydrin group. Since the double bond in the carbon side-chain would allow other reactions to compete with the desired cyclization, we introduced the A ring tertiary 3-OH group, or a function convertible into it before carrying out the cyclization.

Inspired by Krohn's work on the synthesis of angucyclinones, ${ }^{6,7,42}$ we wanted to install a silyl group as a hydroxyl group surrogate at position 3 (Scheme 5). ${ }^{43}$ We first verified the feasibility of the conjugate addition of a silyl cuprate onto a $\beta, \beta$ '-disubstituted enal: Mesityloxide 18 was 
treated with $\left(\mathrm{PhMe}_{2} \mathrm{Si}\right)_{2} \mathrm{CuLi}$ to furnish the expected 3-(dimethylphenylsilanyl)-3-methylbutanal (19) in high yield (Scheme 4).

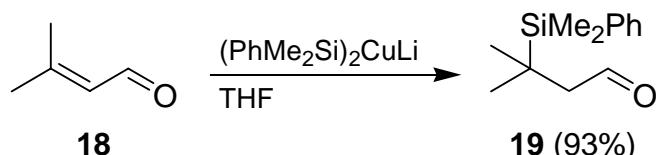

\section{Scheme 4}

However, all attempts to perform similar conjugate additions of silyl cuprates ${ }^{6,7}$ or pentamethyldisilane ${ }^{42}$ onto 8 only led to the recovery of the starting material (Scheme 5). Another option to oxygenate the exocyclic $\mathrm{C}=\mathrm{C}$ double bond of $\mathbf{8}$ was dihydroxylation into diols $21 \mathrm{using}$ catalytic amounts of osmium tetroxide and $N$-methylmorpholine- $N$-oxide (NMO). However, we did not isolate the expected diols 21; instead, two diastereoisomers of an unassigned product, [bis(hemiacetal) 22 or ketal-carbaldehyde hydrate 23] in a combined yield of $78 \%$ were obtained (Scheme 5).

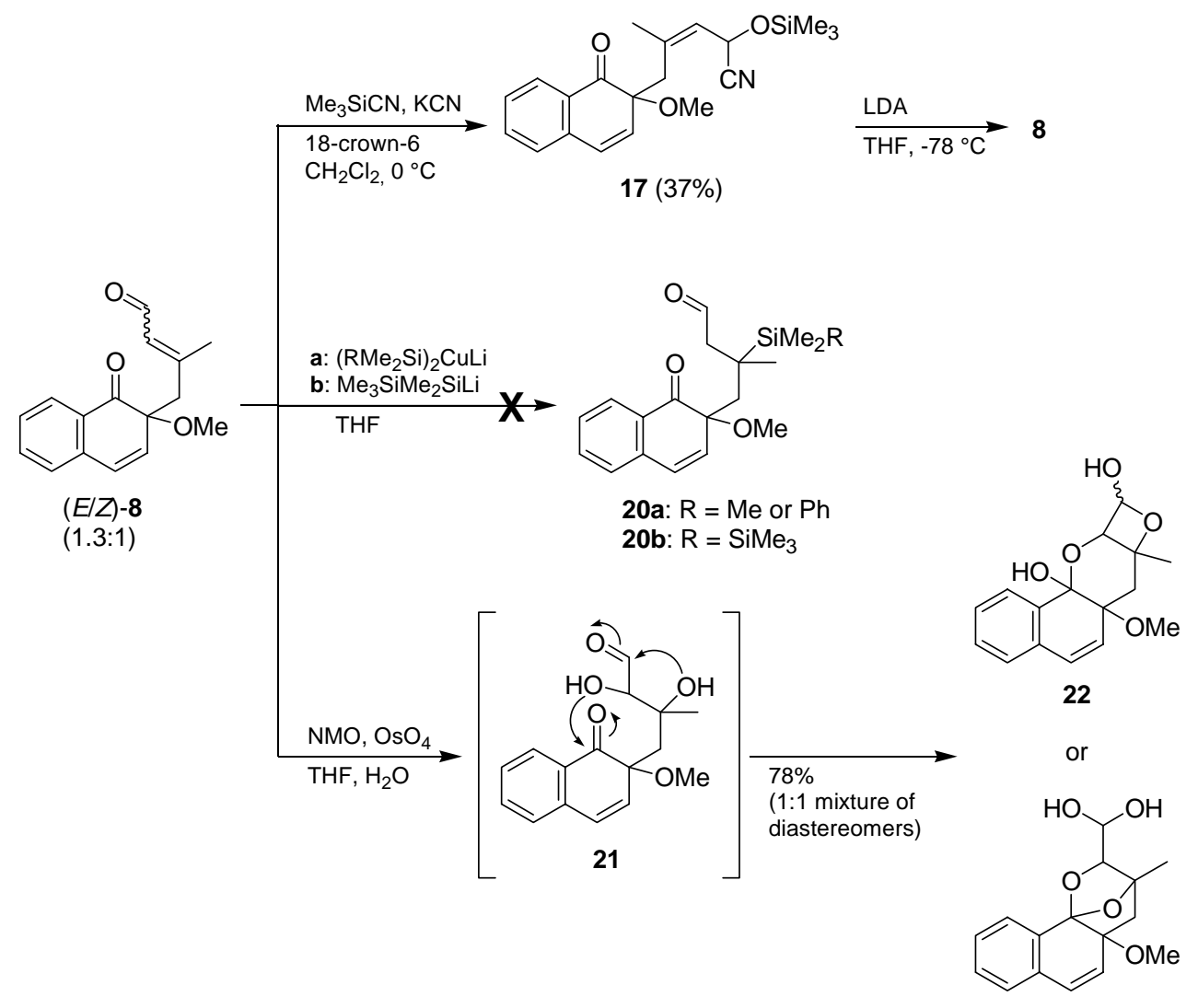

23

\section{Scheme 5}


The most promising approach of introducing only one oxygen atom relied on epoxydation and responded to our expectations in part. Treatment of 8 with $\mathrm{H}_{2} \mathrm{O}_{2}$ in the presence of $\mathrm{Na}_{2} \mathrm{CO}_{3}$ in aqueous $\mathrm{EtOH}^{44}$ afforded the $\alpha, \beta$-epoxyaldehyde 24 (60-65\%) (Scheme 6). This epoxide rapidly decomposed on silica gel, and it was very difficult to obtain it in higher yields with satisfactory purity. Its regioselective opening was accomplished by using the organoselenium reagent developed by Miyashita ${ }^{45}$ to limit dehydration of the $\beta$-hydroxyaldehyde. Treatment of 24 with the phenylseleno(triethyl)borate complex, prepared by reduction of $(\mathrm{PhSe})_{2}$ with $\mathrm{NaBH}_{4}$ in $\mathrm{EtOH}$, gave the five-membered cyclization product 25 (46\%) (Scheme 6). Reductive opening of $\mathbf{2 4}$ using zinc or samarium diiodide furnished only the dehydrated product $\mathbf{2 6}$ in low yield. We treated 25 under mildly basic conditions (i.e., saturated aq. $\mathrm{NaHCO}_{3}$, THF, rt, $19 \mathrm{~h}$ ) with the expectation of converting the five-membered A ring into a six-membered one via a retro-aldol reaction, but the only product isolated was the dehydrated compound $26(15 \%)$, together with recovered starting material 8 (85\%).

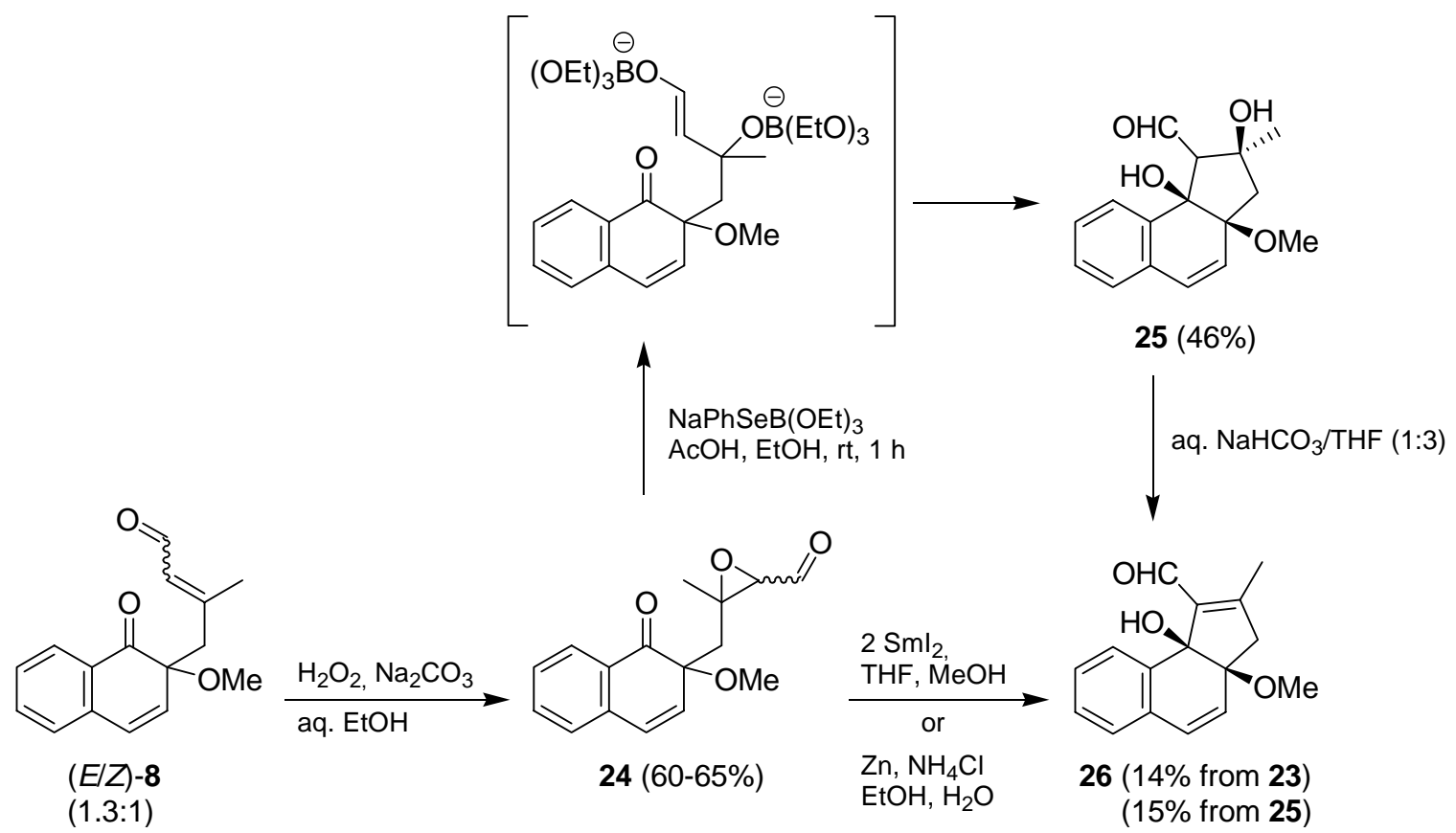

\section{Scheme 6}

Although we were not yet able to generate the aquayamycin-type angucyclinone sixmembered A ring by this approach, the BTI-mediated oxidative nucleophilic substitution of the 2-alkoxynaphthol $\mathbf{9}$ with the silyl enol ether $\mathbf{1 0}$ constitutes a concise route to the highly functionalized naphthoid cyclohexa-2,4-dienone 8. This synthon was transformed into fivemembered ring-containing $\mathrm{ABC}$ models of the angularly-oxygenated benz $[a]$ naphthalene unit of aquayamycin-type angucyclinones. The exploitation of these intermediates in the synthesis of full analogues of aquayamycin (2) is in progress. 


\section{Experimental Section}

General Procedures. Tetrahydrofuran (THF) and diethyl ether were purified by distillation from sodium/ benzophenone under $\mathrm{N}_{2}$ immediately before use. $\mathrm{CH}_{2} \mathrm{Cl}_{2}$ was distilled from $\mathrm{CaH}_{2}$. Light petroleum refers to the $40-60{ }^{\circ} \mathrm{C}$ boiling range. Moisture and oxygen sensitive reactions were carried out in flame-dried glassware under $\mathrm{N}_{2}$. Evaporations were conducted under reduced pressure at temperatures less than $45^{\circ} \mathrm{C}$ unless otherwise noted. Column chromatography was carried out under positive pressure using 40-63 $\mu \mathrm{m}$ silica gel (Merck) and the indicated solvents. Melting points were determined on an Electrothermal IA9100 Digital apparatus. NMR spectra of samples in the indicated solvent were run at 200, 250 or $300 \mathrm{MHz}$. Carbon multiplicities were determined by DEPT-135 experiments. Electron impact mass spectra (EIMS) were obtained at 50-70 eV. Electron impact and liquid secondary ion mass spectrometry at low and high resolution (EIMS, and LSIMS, HRMS) were obtained using a VG-autospec-Q intrument at the CESAMO mass spectrometry laboratory, Université Bordeaux 1.

(2E/Z)-4-(2-Methoxy-1-oxo-1,2-dihydronaphthalen-2-yl)-3-methylbut-2-enal $[(E / Z)-8]$ and (2E)-4-(4-hydroxy-3-methoxy-1-naphthyl)-3-methylbut-2-enal [(E)-11]. To a stirred ice-cold solution of $\mathbf{9}^{39}(1.0 \mathrm{~g}, 5.74 \mathrm{mmol})$ and $\mathbf{1 0}^{46}(2.6 \mathrm{~g}, 16.6 \mathrm{mmol})$ in $\mathrm{CH}_{2} \mathrm{Cl}_{2}(17 \mathrm{~mL})$ was added BTI (4.4 $\mathrm{g}, 10.2 \mathrm{mmol})$ in one portion. After continued stirring at room temperature for $2 \mathrm{~h}$, the mixture was diluted with $\mathrm{CH}_{2} \mathrm{Cl}_{2}(20 \mathrm{~mL})$, washed with saturated aqueous $\mathrm{NaHCO}_{3}(2 \times 20 \mathrm{~mL})$, $1 \mathrm{M} \mathrm{H}_{3} \mathrm{PO}_{4}(20 \mathrm{~mL})$, brine $(20 \mathrm{~mL})$, dried over $\mathrm{Na}_{2} \mathrm{SO}_{4}$, and evaporated. The resulting brownish oil was purified by column chromatography, eluting with light petroleum/ $\mathrm{Et}_{2} \mathrm{O}$ (1:1), to furnish a 1.3:1 E/Z mixture of $8(532 \mathrm{mg}, 36 \%)$ as red oil, and 11 as pale yellow gum (192 $\mathrm{mg}, 13 \%)$.

(E/Z)-8. IR (KBr): 2938, 1670, 1596, $1120 \mathrm{~cm}^{-1}$.

(E)-8: ${ }^{1} \mathrm{H}$ NMR $\left(200 \mathrm{MHz}, \mathrm{CDCl}_{3}\right): \delta 2.20(\mathrm{~s}, 3 \mathrm{H}), 2.57(\mathrm{~s}, 2 \mathrm{H}), 3.16(\mathrm{~s}, 3 \mathrm{H}), 5.74(\mathrm{~d}, J=$ $6.6 \mathrm{~Hz}, 1 \mathrm{H}), 6.08(\mathrm{~d}, J=10.1 \mathrm{~Hz}, 1 \mathrm{H}), 6.76(\mathrm{~d}, J=10.1 \mathrm{~Hz}, 1 \mathrm{H}), 7.23(\mathrm{dd}, J=1.2,7.6 \mathrm{~Hz}, 1 \mathrm{H})$, $7.37(\mathrm{~m}, 1 \mathrm{H}), 7.58(\mathrm{~m}, 1 \mathrm{H}), 7.98(\mathrm{~m}, 1 \mathrm{H}), 9.89(\mathrm{~d}, J=7.9 \mathrm{~Hz}, 1 \mathrm{H}) ;{ }^{13} \mathrm{C} \mathrm{NMR}(62.9 \mathrm{MHz}$, $\left.\mathrm{CDCl}_{3}\right): \delta 199.5,190.8,157.8,136.9,135.3,134.8,131.3,129.8,129.6,128.7,127.9,127.2$, 82.3, 53.7, 49.6, 19.7 .

(Z)-8. ${ }^{1} \mathrm{H}$ NMR $\left(300 \mathrm{MHz}, \mathrm{CDCl}_{3}\right): \delta 1.93(\mathrm{~s}, 3 \mathrm{H}), 2.79(\mathrm{~d}, J=13.2 \mathrm{~Hz}, 1 \mathrm{H}), 2.91(\mathrm{~d}, J=$ $13.2 \mathrm{~Hz}, 1 \mathrm{H}), 3.09(\mathrm{~s}, 3 \mathrm{H}), 5.69(\mathrm{~d}, J=8.6 \mathrm{~Hz}, 1 \mathrm{H}), 6.02(\mathrm{~d}, J=9.8 \mathrm{~Hz}, 1 \mathrm{H}), 6.71(\mathrm{~d}, J=9.8 \mathrm{~Hz}$, 1H), $7.17(\mathrm{~m}, 1 \mathrm{H}), 7.30(\mathrm{~m}, 1 \mathrm{H}), 7.52(\mathrm{~m}, 1 \mathrm{H}), 7.92(\mathrm{~d}, J=7.5 \mathrm{~Hz}, 1 \mathrm{H}), 9.61(\mathrm{~d}, J=7.9 \mathrm{~Hz}, 1 \mathrm{H})$; ${ }^{13} \mathrm{C}$ NMR $\left(75.5 \mathrm{MHz}, \mathrm{CDCl}_{3}\right): \delta 199.4,190.7,156.8,136.7,135.3,134.8,131.3,129.7,129.5$, 128.7, 127.9, 127.1, 82.0, 53.7, 41.3, 27.7. LSIMS: $\mathrm{m} / \mathrm{z}$ (rel intensity) 279 (58) $\left[\mathrm{MNa}^{+}\right], 257$ (41) $\left[\mathrm{MH}^{+}\right], 256$ (35) $\left[\mathrm{M}^{+}\right], 225$ (59); HMRS (LSIMS): Calcd for $\mathrm{C}_{16} \mathrm{H}_{16} \mathrm{O}_{3}$ 256.1099. Found 256.1091 .

(E)-11. IR (KBr): 3292, $1649 \mathrm{~cm}^{-1} ;{ }^{1} \mathrm{H}$ NMR (250 MHz, $\left.\mathrm{CDCl}_{3}\right): \delta 2.20(\mathrm{~s}, 3 \mathrm{H}), 3.88(\mathrm{~s}, 2 \mathrm{H})$, 3.94 (s, 3H), 5.75 (br d, $J=8.1 \mathrm{~Hz}, 1 \mathrm{H}), 6.20(\mathrm{~s}, 1 \mathrm{H}), 7.07(\mathrm{~s}, 1 \mathrm{H}), 7.41(\mathrm{~m}, 2 \mathrm{H}), 7.73(\mathrm{~d}, J=$ $8.1 \mathrm{~Hz}, 1 \mathrm{H}), 8.20(\mathrm{~d}, J=7.9 \mathrm{~Hz}, 1 \mathrm{H}), 9.98(\mathrm{~d}, J=7.9 \mathrm{~Hz}, 1 \mathrm{H}) ;{ }^{13} \mathrm{C} \mathrm{NMR}\left(62.9 \mathrm{MHz}, \mathrm{CDCl}_{3}\right): \delta$ $191.4,162.9,140.6,139.4,131.9,128.3,127.9,125.4,124.8,124.6,123.6,122.0,115.6,57.3$, 
43.7, 17.8; LSIMS: m/z (rel intensity) 279 (18) $\left[\mathrm{MNa}^{+}\right], 256$ (100) $\left[\mathrm{M}^{+}\right]$; HMRS (LSIMS): Calcd for $\mathrm{C}_{16} \mathrm{H}_{16} \mathrm{O}_{3} 256.1099$. Found 256.1098.

2-(4-Hydroxy-3-methoxy-1-naphthyl)-3-methylbut-2-enal (16). 3-Benzyl-5-(2-hydroxyethyl)4-methyl-1,3-thiazolium chloride $\left(\mathbf{1 2}, 1.4 \mathrm{mg}, 0.005 \mathrm{mmol}^{2}\right)$ and $\mathrm{Et}_{3} \mathrm{~N}(4 \mu \mathrm{L}, 0.03 \mathrm{mmol})$ were successively added to a solution of $8(25 \mathrm{mg}, 0.098 \mathrm{mmol})$ in absolute ethanol $(2 \mathrm{~mL})$. The reaction mixture was heated at $80{ }^{\circ} \mathrm{C}$ for $2.5 \mathrm{~h}$, after which time ethanol was evaporated. The residue was then diluted with $\mathrm{CH}_{2} \mathrm{Cl}_{2}(10 \mathrm{~mL})$, washed with saturated aqueous $\mathrm{Na}_{2} \mathrm{CO}_{3}(10 \mathrm{~mL})$, brine $(20 \mathrm{~mL})$, dried over $\mathrm{Na}_{2} \mathrm{SO}_{4}$, and evaporated. The resulting crude oil was purified by column chromatography, eluting with light petroleum/ $\mathrm{Et}_{2} \mathrm{O}(1: 1)$, to give $\mathbf{1 6}$ as light red gum (23 mg, 90\%). IR (NaCl) 3408, 2928, 1694, $1350 \mathrm{~cm}^{-1} ;{ }^{1} \mathrm{H}$ NMR (250 MHz, $\left.\mathrm{CDCl}_{3}\right): \delta 1.71$ (s, $3 \mathrm{H}), 2.47(\mathrm{~s}, 3 \mathrm{H}), 3.97(\mathrm{~s}, 3 \mathrm{H}), 6.03(\mathrm{~s}, 1 \mathrm{H}), 6.94(\mathrm{~s}, 1 \mathrm{H}), 7.30(\mathrm{~d}, J=8.3 \mathrm{~Hz}, 1 \mathrm{H}), 7.42(\mathrm{~m}, 2 \mathrm{H})$, $8.17(\mathrm{~d}, J=7.6 \mathrm{~Hz}, 1 \mathrm{H}), 10.36(\mathrm{~s}, 1 \mathrm{H}) ;{ }^{13} \mathrm{C} \mathrm{NMR}\left(62.9 \mathrm{MHz}, \mathrm{CDCl}_{3}\right): \delta 190.7,158.7,140.8$, 139.5, 137.1, 128.0, 125.8, 125.4, 124.8, 124.7, 124.3, 121.8, 115.0, 57.2, 25.3, 19.9; LSIMS: $\mathrm{m} / \mathrm{z}$ (rel intensity) 279 (28) $\left[\mathrm{MNa}^{+}\right], 256$ (100) $\left[\mathrm{M}^{+}\right]$; HMRS (LSIMS): Calcd for $\mathrm{C}_{16} \mathrm{H}_{16} \mathrm{O}_{3}$ 256.1099. Found 256.1101.

(3Z)-5-(2-Methoxy-1-oxo-1,2-dihydronaphthalen-2-yl)-4-methyl-2-[(trimethylsilyl)oxy] hyphen: pent3-enenitrile (17). To a stirred solution of $8(150 \mathrm{mg}, 0.59 \mathrm{mmol}), \mathrm{KCN}(3 \mathrm{mg}, 0.08 \mathrm{mmol})$ and 18 crown-6 (8 mg, $0.05 \mathrm{mmol})$ in $\mathrm{CH}_{2} \mathrm{Cl}_{2}(6 \mathrm{~mL})$ was added dropwise at $0{ }^{\circ} \mathrm{C} \mathrm{Me}_{3} \mathrm{SiCN}(94 \mu 1$, $0.71 \mathrm{mmol}$ ). The reaction mixture was stirred at $0^{\circ} \mathrm{C}$ for $45 \mathrm{~min}$; after evaporation of the solvent the residue was submitted to column chromatography, eluting with light petroleum/ $\mathrm{Et}_{2} \mathrm{O}(2: 1)$ to give 17 as a pale yellow oil $(77 \mathrm{mg}, 37 \%) .{ }^{1} \mathrm{H} \mathrm{NMR}\left(250 \mathrm{MHz}, \mathrm{CDCl}_{3}\right): \delta 0.16(\mathrm{~s}, 9 \mathrm{H}), 1.67(\mathrm{~s}$, $3 \mathrm{H}), 1.79(\mathrm{~s}, 3 \mathrm{H}), 2.46(\mathrm{~s}, 2 \mathrm{H}), 2.50(\mathrm{~s}, 2 \mathrm{H}), 3.17(\mathrm{~s}, 3 \mathrm{H}), 3.18(\mathrm{~s}, 3 \mathrm{H}), 4.93(\mathrm{~d}, J=8.5,1 \mathrm{H}), 4.99$ $(\mathrm{d}, J=7.9 \mathrm{~Hz}, 1 \mathrm{H}), 5.26(\mathrm{~d}, J=8.5 \mathrm{~Hz}, 1 \mathrm{H}), 5.32$ (d, $J=7.3 \mathrm{~Hz}, 1 \mathrm{H}), 6.09$ (d, J=10.1 Hz, 1H), $6.79(\mathrm{~d}, J=10.1 \mathrm{~Hz}, 1 \mathrm{H}), 7.24(\mathrm{~d}, J=7.6 \mathrm{~Hz}, 1 \mathrm{H}), 7.37$ (t, $J=7.6 \mathrm{~Hz}, 1 \mathrm{H}), 7.59$ (t, $J=7.6 \mathrm{~Hz}$, $1 \mathrm{H}), 8.02(\mathrm{~d}, J=7.6 \mathrm{~Hz}, 1 \mathrm{H}) ;{ }^{13} \mathrm{C} \mathrm{NMR}\left(62.9 \mathrm{MHz}, \mathrm{CDCl}_{3}\right): \delta 200.0,137.1,136.9,135.0,135.0$, 134.9 , 134.9, 129.9, 129.3, 129.1, 128.3, 126.9, 126.8, 125.8, 82.3, 57.9, 53.4, 48.6, 48.3, 18.7, 18.5, -0.4; EIMS: $\mathrm{m} / \mathrm{z}$ (rel intensity) 355 (7) $\left[\mathrm{M}^{+}\right]$; HMRS (EIMS): Calcd for $\mathrm{C}_{20} \mathrm{H}_{25} \mathrm{NO}_{3} \mathrm{Si}$ 355.1603. Found 355.1601.

3-[Dimethyl(phenyl)silyl]-3-methylbutanal (19). Dimethylphenylsilyl lithium in THF (0.48 M, $2.3 \mathrm{~mL}, 1.1 \mathrm{mmol}$ ) was added dropwise to a suspension of copper iodide $(105 \mathrm{mg}, 0.55 \mathrm{mmol})$ in THF $(1 \mathrm{~mL})$ at $-78^{\circ} \mathrm{C}$. The reaction mixture was stirred at $-25^{\circ} \mathrm{C}$ for $30 \mathrm{~min}$, recooled to $-78^{\circ} \mathrm{C}$, and the mesityloxide $(18,42 \mathrm{mg}, 0.5 \mathrm{mmol})$ was added. After stirring at $-60{ }^{\circ} \mathrm{C}$ for $2.5 \mathrm{~h}$, the reaction was quenched with saturated aqueous $\mathrm{NH}_{4} \mathrm{Cl}(10 \mathrm{~mL})$ and extracted with $\mathrm{Et}_{2} \mathrm{O}(2 \times 10 \mathrm{~mL})$. The combined organic layers were washed with $10 \% \mathrm{HCl}(5 \mathrm{~mL})$, brine $(10 \mathrm{~mL})$, dried over $\mathrm{Na}_{2} \mathrm{SO}_{4}$, and evaporated. The resulting oil was purified by column chromatography, eluting with light petroleum/Et $2 \mathrm{O}(6: 1)$, to afford $19(102 \mathrm{mg}, 93 \%)$. IR (NaCl) 2958, 2866, 1717, 1468, $1111 \mathrm{~cm}^{-1}$; ${ }^{1} \mathrm{H} \mathrm{NMR}$ $\left(250 \mathrm{MHz}, \mathrm{CDCl}_{3}\right): \delta 0.33(\mathrm{~s}, 6 \mathrm{H}), 1.11(\mathrm{~s}, 6 \mathrm{H}), 2.23(\mathrm{~d}, J=3.3 \mathrm{~Hz}, 2 \mathrm{H}), 7.38(\mathrm{~m}, 3 \mathrm{H}), 7.48(\mathrm{~m}$, 2H), $9.78(\mathrm{t}, J=3.3 \mathrm{~Hz}, 1 \mathrm{H}) ;{ }^{13} \mathrm{C} \mathrm{NMR}\left(62.9 \mathrm{MHz}, \mathrm{CDCl}_{3}\right): \delta 204.5,134.5,129.3,127.7,51.7$, $23.4,-6.1$. 


\section{6a-Methoxy-7a-methyl-7,7a,9,9a-tetrahydrobenzo[h]oxeto[3,2-b]chromene-9,10a(6aH)- diol}

(22) or (4a-methoxy-3-methyl-2,3,4,4a-tetrahydro-3,10b-epoxybenzo[h]chromen-2-yl) methanediol (23). To a solution of 8 (160 mg, $0.62 \mathrm{mmol})$ in THF $(6 \mathrm{~mL})$ and $\mathrm{H}_{2} \mathrm{O}(1 \mathrm{~mL})$ were added at $\mathrm{rt} \mathrm{OsO}_{4}(7.9 \mathrm{mg}, 0.03 \mathrm{mmol})$ and $\mathrm{NMO}(210 \mathrm{mg}, 1.56 \mathrm{mmol})$. After $24 \mathrm{~h}$ the mixture was treated with aqueous $\mathrm{Na}_{2} \mathrm{~S}_{2} \mathrm{O}_{3}$ and stirred for $40 \mathrm{~min}$, after which time it was extracted with EtOAc $(2 \times 10 \mathrm{~mL})$. The combined organic layers were washed with brine $(10 \mathrm{~mL})$, dried over $\mathrm{Na}_{2} \mathrm{SO}_{4}$, and evaporated. The resulting oil was purified by column chromatography, eluting with light petroleum/acetone (2:1), to furnish two separate but unassigned diastereoisomers as yellow oils, the faster moving isomer 22a or 23a (69 mg, 38\%) and the slower one 22b or $\mathbf{2 3 b}$ (72 $\mathrm{mg}$, $40 \%$ ).

22a/23a. IR (KBr): 3420 (br), 2930, 1469, $1064 \mathrm{~cm}^{-1} .{ }^{1} \mathrm{H}$ NMR $\left(250 \mathrm{MHz}, \mathrm{CDCl}_{3}\right): \delta 1.34$ (s, $3 \mathrm{H}), 2.10(\mathrm{~d}, J=13.7 \mathrm{~Hz}, 1 \mathrm{H}), 2.62(\mathrm{~d}, J=8.5 \mathrm{~Hz}, 1 \mathrm{H}), 3.04(\mathrm{~s}, 3 \mathrm{H}), 3.48(\mathrm{~d}, J=7.2 \mathrm{~Hz}, 1 \mathrm{H})$, $5.62(\mathrm{~d}, J=7.2 \mathrm{~Hz}, 1 \mathrm{H}), 5.73(\mathrm{~d}, J=9.7 \mathrm{~Hz}, 1 \mathrm{H}), 6.67(\mathrm{~d}, J=9.7 \mathrm{~Hz}, 1 \mathrm{H}), 7.26(\mathrm{~m}, 1 \mathrm{H}), 7.34$ (m, 2H), $7.89(\mathrm{~m}, 1 \mathrm{H}) ;{ }^{13} \mathrm{C} \mathrm{NMR}\left(62.9 \mathrm{MHz}, \mathrm{CDCl}_{3}\right): \delta 132.7,131.0,129.4,129.3,128.4,127.9$, 127.3, 125.8, 101.6, 95.5, 81.9, 79.0, 75.2, 52.0, 44.9, 22.8; LSIMS: $\mathrm{m} / \mathrm{z}$ (rel intensity) 313 (100) [MNa ${ }^{+}$; HMRS (LSIMS): Calcd for $\mathrm{C}_{16} \mathrm{H}_{18} \mathrm{O}_{5} \mathrm{Na} 313.1052$. Found 313.1058.

22b/23b. mp $138{ }^{\circ} \mathrm{C}$, IR (KBr): 3414 (br), 2926, 1706, $1066 \mathrm{~cm}^{-1} ;{ }^{1} \mathrm{H}$ NMR (250 MHz, acetone$\left.d_{6}\right): \delta 1.35(\mathrm{~s}, 3 \mathrm{H}), 1.93(\mathrm{~d}, J=13.1 \mathrm{~Hz}, 1 \mathrm{H}), 2.18(\mathrm{~d}, J=8.5 \mathrm{~Hz}, 1 \mathrm{H}), 2.98(\mathrm{~s}, 3 \mathrm{H}), 3.75(\mathrm{~d}, J=$ $2.1 \mathrm{~Hz}, 1 \mathrm{H}), 5.85(\mathrm{~s}, 1 \mathrm{H}), 6.02(\mathrm{~d}, J=9.7 \mathrm{~Hz}, 1 \mathrm{H}), 6.67(\mathrm{~d}, J=9.7 \mathrm{~Hz}, 1 \mathrm{H}), 7.18(\mathrm{~m}, 1 \mathrm{H}), 7.30$ $(\mathrm{m}, 2 \mathrm{H}), 7.73(\mathrm{~m}, 1 \mathrm{H}) ;{ }^{13} \mathrm{C}$ NMR (acetone- $\left.d_{6}, 62.9 \mathrm{MHz}\right): \delta 135.1,134.8,132.0,131.2,129.8$, 128.6, 127.3, 126.0, 108.5, 97.0, 87.2, 73.6, 67.6, 50.3, 44.1, 27.3; LSIMS: $\mathrm{m} / \mathrm{z}$ (rel intensity) 313 (100) $\left[\mathrm{MNa}^{+}\right]$; HMRS (LSIMS): Calcd for $\mathrm{C}_{16} \mathrm{H}_{18} \mathrm{O}_{5} \mathrm{Na} 313.1052$. Found 313.1059.

3-[(2-Methoxy-1-oxo-1,2-dihydronaphthalen-2-yl)methyl]-3-methyloxirane-2-carbaldehyde (24). To a suspension of $8(120 \mathrm{mg}, 0.47 \mathrm{mmol})$ and $\mathrm{Na}_{2} \mathrm{CO}_{3}(49.8 \mathrm{mg}, 0.47 \mathrm{mmol})$ in $\mathrm{EtOH} / \mathrm{H}_{2} \mathrm{O}(4: 1$, $7 \mathrm{~mL})$ was added dropwise at $0{ }^{\circ} \mathrm{C}$ an aqueous solution of $\mathrm{H}_{2} \mathrm{O}_{2}(30 \%, 400 \mu \mathrm{L})$. After the addition was complete, the ice-bath was removed, and the mixture was stirred at room temperature for $3 \mathrm{~h}$. The mixture was then evaporated, and the residue was diluted with $\mathrm{CH}_{2} \mathrm{Cl}_{2}$ $(10 \mathrm{~mL})$, washed with $\mathrm{H}_{2} \mathrm{O}(10 \mathrm{~mL})$, brine $(10 \mathrm{~mL})$, dried over $\mathrm{Na}_{2} \mathrm{SO}_{4}$, and evaporated at $\mathrm{rt}$. The resulting oil was purified by column chromatography, eluting with ligth petroleum/Et ${ }_{2} \mathrm{O}(2: 3)$, to afford a 1:1 mixture of epoxide diastereosomers 24 as a light yellow oil (85 $\mathrm{mg}, 67 \%$ ). Preparative TLC, eluting with light petroleum/acetone (3:1), furnished 24a as the faster moving isomer and 24b.

24a. ${ }^{1} \mathrm{H}$ NMR (250 MHz, $\left.\mathrm{CDCl}_{3}\right): \delta 1.42(\mathrm{~s}, 3 \mathrm{H}), 2.10(\mathrm{~d}, J=14.6 \mathrm{~Hz}, 1 \mathrm{H}), 2.31(\mathrm{~d}, J=14.6 \mathrm{~Hz}$, $1 \mathrm{H}), 3.18(\mathrm{~s}, 3 \mathrm{H}), 6.13(\mathrm{~d}, J=10.0 \mathrm{~Hz}, 1 \mathrm{H}), 6.72(\mathrm{~d}, J=10.0 \mathrm{~Hz}, 1 \mathrm{H}), 7.24(\mathrm{~d}, J=7.9 \mathrm{~Hz}, 1 \mathrm{H})$, $7.39(\mathrm{~m}, 1 \mathrm{H}), 7.60(\mathrm{t}, J=1.5,7.8 \mathrm{~Hz}, 1 \mathrm{H}), 7.99$ (d, $J=8.5 \mathrm{~Hz}, 1 \mathrm{H}), 9.28(\mathrm{~d}, J=5.2 \mathrm{~Hz}, 1 \mathrm{H})$; EIMS: $m / z$ (rel intensity) $272\left(\mathrm{M}^{+}, 15\right), 157$ (100).

24b. ${ }^{1} \mathrm{H} \mathrm{NMR}\left(\mathrm{CDCl}_{3}, 250 \mathrm{MHz}\right) \delta 1.48(\mathrm{~s}, 3 \mathrm{H}), 1.88(\mathrm{~d}, J=14.6 \mathrm{~Hz}, 1 \mathrm{H}), 2.20(\mathrm{~d}, J=14.6 \mathrm{~Hz}$, $1 \mathrm{H}), 3.18(\mathrm{~s}, 3 \mathrm{H}), 6.18(\mathrm{~d}, J=10.0 \mathrm{~Hz}, 1 \mathrm{H}), 6.82(\mathrm{~d}, J=10.0 \mathrm{~Hz}, 1 \mathrm{H}), 7.24(\mathrm{~d}, J=6.1 \mathrm{~Hz}, 1 \mathrm{H})$, $7.39(\mathrm{~m}, 1 \mathrm{H}), 7.60(\mathrm{t}, J=1.5,7.8 \mathrm{~Hz}, 1 \mathrm{H}), 8.03(\mathrm{~d}, J=8.5 \mathrm{~Hz}, 1 \mathrm{H}), 9.37$ (d, $J=4.9 \mathrm{~Hz}, 1 \mathrm{H})$; EIMS: $m / z$ (rel intensity) $272(15)\left[\mathrm{M}^{+}\right], 157$ (100). 
(3aS*,9b $R^{*}$ )-2,9b-Dihydroxy-3a-methoxy-2-methyl-2,3,3a,9b-tetrahydro-1H-cyclopenta[a]naphthalene-1-carbaldehyde (25). $\mathrm{NaBH}_{4}(38 \mathrm{mg}, 1.00 \mathrm{mmol})$ was added portionwise to a solution of diphenyldiselenide $(160 \mathrm{mg}, 0.51 \mathrm{mmol})$ in absolute EtOH $(2 \mathrm{~mL})$. Once the gas evolution ceased, the yellow solution was cooled to $0{ }^{\circ} \mathrm{C}$ using an ice-water bath, treated with $\mathrm{AcOH}(9.8 \mu \mathrm{L})$, and then added at $\mathrm{rt}$ to a solution of epoxide $24(90 \mathrm{mg}, 0.33 \mathrm{mmol})$ in EtOH $(2 \mathrm{~mL})$. The reaction mixture turned blue and was stirred for $2.5 \mathrm{~h}$, after which time it was diluted with EtOAc and bubbled with oxygen gas for several minutes to convert the remaining selenium reagent to $(\mathrm{PhSe})_{2}$. The organic layer was washed with brine $(10 \mathrm{~mL})$, dried over $\mathrm{Na}_{2} \mathrm{SO}_{4}$, and evaporated at $\mathrm{rt}$. The resulting crude oil was purified by column chromatography, eluting with ligth petroleum/acetone (3:1), to furnish 25 as a yellow oil (42 $\mathrm{mg}, 46 \%)$ : IR ( $\mathrm{KBr}$ ) 3447, 2933, $1716 \mathrm{~cm}^{-1}$; ${ }^{1} \mathrm{H}$ NMR $\left(250 \mathrm{MHz}, \mathrm{CDCl}_{3}\right): \delta 1.39(\mathrm{~s}, 3 \mathrm{H}), 2.40(\mathrm{~d}, J=14.9 \mathrm{~Hz}, 1 \mathrm{H})$, $2.54(\mathrm{~d}, J=2.1 \mathrm{~Hz}, 1 \mathrm{H}), 2.58(\mathrm{~d}, J=15.5 \mathrm{~Hz}, 1 \mathrm{H}), 3.08(\mathrm{~s}, 3 \mathrm{H}), 3.88(\mathrm{~s}, 1 \mathrm{H}), 3.88(\mathrm{~s}, 1 \mathrm{H}), 4.08$ $(\mathrm{s}, 1 \mathrm{H}), 5.75(\mathrm{~d}, J=9.8 \mathrm{~Hz}, 1 \mathrm{H}), 6.73(\mathrm{~d}, J=9.8 \mathrm{~Hz}, 1 \mathrm{H}), 7.16(\mathrm{~d}, J=7.1 \mathrm{~Hz}, 1 \mathrm{H}), 7.26-7.30(\mathrm{~m}$, 2H), 7.70 (d, $J=7.5 \mathrm{~Hz}, 1 \mathrm{H}), 9.90$ (d, $J=2.5 \mathrm{~Hz}, 1 \mathrm{H}) ;{ }^{13} \mathrm{C}$ NMR $\left(62.9 \mathrm{MHz}, \mathrm{CDCl}_{3}\right): \delta 203.3$, 137.7, 131.2, 129.9, 129.1, 128.6, 128.2, 127.8, 125.1, 82.4, 80.3, 78.1, 66.3, 54.8, 52.0, 28.2; LSIMS: m/z (rel intensity) 297 (MNa $\left.{ }^{+}, 100\right)$; HMRS (LSIMS): Calcd for $\mathrm{C}_{16} \mathrm{H}_{18} \mathrm{O}_{4} \mathrm{Na} 297.1103$. Found 297.1106.

(3aS*,9bS*)-9b-Hydroxy-3a-methoxy-2-methyl-3a,9b-dihydro-3H-cyclopenta[a]

naphthalene-1carbaldehyde (26). A suspension of 24 (17 mg, $0.06 \mathrm{mmol})$, zinc powder (20 $\mathrm{mg}, 0.31 \mathrm{mmol})$, ammonium chloride $(10 \mathrm{mg}, 0.18 \mathrm{mmol})$ in a $4: 1$ mixture of $\mathrm{EtOH} / \mathrm{H}_{2} \mathrm{O}(7 \mathrm{~mL})$ was heated at $80{ }^{\circ} \mathrm{C}$ for $20 \mathrm{~min}$, after which time it was filtered. The filtration pellet was rinsed with $\mathrm{Et}_{2} \mathrm{O}$, and the filtrates were washed with brine $(10 \mathrm{~mL})$, dried over $\mathrm{Na}_{2} \mathrm{SO}_{4}$ and evaporated at $\mathrm{rt}$. The residue was purified by PLC, eluting with light petroleum/ $\mathrm{Et}_{2} \mathrm{O}(2: 3)$, to afford 26 as yellow oil (17 mg, 14\%). IR (KBr) 3430, 2930, 1680, 1608, $753 \mathrm{~cm}^{-1} ;{ }^{1} \mathrm{H}$ NMR $\left(250 \mathrm{MHz}, \mathrm{CDCl}_{3}\right): \delta 2.19$ (s, 3H), $2.63(\mathrm{~d}, J=17.4 \mathrm{~Hz}, 1 \mathrm{H}), 3.12(\mathrm{~d}, J=17.4 \mathrm{~Hz}, 1 \mathrm{H}), 3.17$ (s, 3H), $3.48(\mathrm{~s}, 1 \mathrm{H}), 5.73$ (d, $J$ $=9.8 \mathrm{~Hz}, 1 \mathrm{H}), 6.73(\mathrm{~d}, J=9.8 \mathrm{~Hz}, 1 \mathrm{H}), 7.25-7.28(\mathrm{~m}, 1 \mathrm{H}), 7.32-7.36(\mathrm{~m}, 1 \mathrm{H}), 7.95(\mathrm{~d}, J=$ $7.6 \mathrm{~Hz}, 1 \mathrm{H}), 9.51$ (s, $1 \mathrm{H}) ;{ }^{13} \mathrm{C} \mathrm{NMR}\left(62.9 \mathrm{MHz}, \mathrm{CDCl}_{3}\right): \delta 189.4,160.9,136.6,136.3,132.7$, $130.4,128.7,128.1,127.3,127.1,126.9,80.9,80.2,52.3,49.8,16.4$; EIMS: $\mathrm{m} / \mathrm{z}$ (rel intensity) 256 (55) $\left[\mathrm{M}^{+}\right], 224$ (100), 195 (73); HMRS (EIMS): Calcd for $\mathrm{C}_{16} \mathrm{H}_{16} \mathrm{O}_{3}$ 256.1099. Found 256.1103.

\section{Acknowledgements}

The support of the Centre National de la Recherche Scientifique (French part of the IGERT Program) is gratefully acknowledged. The authors also wish to thank the Ministère délégué à la Recherche et aux Nouvelles Technologies for G.-J.F.'s postdoctoral fellowship and for N.L.'s graduate research assistantship (N.L. was awarded with this assistantship through the French part of the IGERT Program) and Simafex (www.simafex.com) for the gift of BTI reagent. 


\section{References}

1. Rohr, J.; Thiericke, R. Nat. Prod. Rep. 1992, 9, 103.

2. Krohn, K.; Rohr, J. Top. Curr. Chem. 1997, 188, 127.

3. Guingant, A.; Barreto, M. M. Tetrahedron Lett. 1987, 28, 3107.

4. Larsen, D. S.; O'Shea, M. D. J. Chem. Soc., Perkin Trans I 1995, 1019.

5. Larsen, D. S.; O'Shea, M. D.; Brooker, S. Chem. Comm. 1996, 203.

6. Krohn, K.; Khanbabaee, K. Liebigs Ann. Chem. 1994, 1109.

7. Krohn, K.; Khanbabaee, K.; Micheel, J. Liebigs Ann. Chem. 1995, 1529.

8. Carreño, C. M.; Urbano, A.; Di Vitta, C. Chem. Comm. 1999, 817.

9. Kim, K.; Boyd, V. A.; Sobti, A.; Sulikowski, G. A. Isr. J. Chem. 1997, 37, 3.

10. Matsumoto, T.; Sohma, T.; Yamaguchi, H.; Kurata, S.; Suzuki, K. Tetrahedron 1995, 51, 7347.

11. Boyd, V. A.; Sulikowski, G. A. J. Am. Chem. Soc. 1995, 117, 8472.

12. Landells, J. S.; Larsen, D. S.; Simpson, J. Tetrahedron Lett. 2003, 44, 5193.

13. Katsuura, K.; Snieckus, V. Tetrahedron Lett. 1985, 26, 9.

14. Katsuura, K.; Snieckus, V. Can. J. Chem. 1987, 65, 124.

15. Uemura, M.; Nishikawa, N.; Take, K.; Ohnishi, M.; Hirotsu, K.; Higushi, T.; Hayashi, Y. J. Org. Chem. 1983, 48, 2349.

16. Gordon, D. M.; Danishefsky, S. J. J. Org. Chem. 1992, 57, 7052.

17. Mitchell, A. S.; Russell, R. A. Tetrahedron 1997, 53, 4387.

18. Chuang, C.-P.; Wang, S.-V. Tetrahedron Lett. 1994, 35, 4365.

19. Brown, P. M.; Thomson, R. H. J. Chem. Soc., Perkin Trans. I 1976, 997.

20. Krohn, K.; Böker, N.; Flörke, U.; Freund, C. J. Org. Chem. 1997, 62, 2350.

21. Yamaguchi, M.; Okuma, T.; Horiguchi, A.; Ikeura, C.; Minami, T. J. Org. Chem. 1992, 57, 1647.

22. Kraus, G. A.; Wan, Z. Tetrahedron Lett. 1997, 38, 6509.

23. Nagatsu, T.; Ayukawa, S.; Umezawa, K. J. Antibiot. 1968, 21, 354.

24. Ayukawa, S.; Takeuchi, T.; Sezaki, M.; Hara, T.; Umezawa, K.; Nagatsu, T. J. Antibiotics 1968, $21,350$.

25. Hayaishi, O.; Okuno, S.; Fujisawa, H. Biochem. Biophys. Res. Commun. 1970, 39, 643.

26. Nozaki, M.; Okuno, S.; Fujisawa, H. Biochem. Biophys. Res. Commun. 1971, 44, 1109.

27. Tanaka, N.; Okabe, T.; Tanaka, N.; Take, K.; Inouye, Y.; Nakamura, S.; Nakashima, H.; Yamamoto, Y. Jpn. J. Cancer. Res. 1986, 77, 324.

28. Henkel, T.; Zeeck, A. J. Antibiot. 1990, 43, 830.

29. Sekisawa, R.; Iinuma, H.; Naganawa, H.; Hamada, S.; Takeuchi, T.; Yamaizumi, J.; Umezawa, K. J. Antibiotics 1996, 49, 487.

30. Uchida, T.; Imoto, M.; Watanabe, Y.; Miura, K.; Dobashi, T.; Matsuda, N.; Sawa, T.; Naganawa, H.; Hamada, M.; Takeuchi, T.; Umezawa, H. J. Antibiot. 1985, 38, 1171.

31. Okazaki, T.; Kitahara, T.; Okami, Y. J. Antibiot. 1975, 28, 176. 
32. Omura, S.; Tanaka, H.; Oiwa, R.; Awaya, J.; Masuma, R.; Tanaka, K. J. Antibiot. 1977, 30, 908.

33. Kitahara, T.; Naganawa, H.; Okazaki, T.; Okami, Y.; Umezawa, H. J. Antibiot. 1975, 28, 280.

34. Krohn, K.; Frese, P.; Flörke, U. Chem. Eur. J. 2000, 6, 3887.

35. Matsumoto, T.; Yamaguchi, H.; Hamura, T.; Tanabe, M.; Kuriyama, Y.; Suzuki, K. Tetrahedron Lett. 2000, 41, 8383.

36. Matsumoto, T.; Yamaguchi, H.; Tanabe, M.; Yasui, Y.; Suzuki, K. Tetrahedron Lett. 2000, 41, 8393.

37. Yamaguchi, H.; Konegawa, T.; Tanabe, M.; Nakamura, T.; Matsumoto, T.; Suzuki, K. Tetrahedron Lett. 2000, 41, 8389.

38. Quideau, S.; Looney, M. A.; Pouységu, L. Org. Lett. 1999, 1, 1651.

39. Quideau, S.; Pouységu, L.; Oxoby, M.; Looney, M. A. Tetrahedron 2001, 57, 319.

40. Stetter, H. Angew. Chem. Int. Ed. Engl. 1976, 15, 639.

41. Hachishu, Y.; Bode, J. W.; Suzuki, K. J. Am. Chem. Soc. 2003, 125, 8432.

42. Krohn, K.; Khanbabaee, K. Angew. Chem. Int. Ed. Engl. 1994, 33, 99.

43. Lipshutz, B. H.; Sclafani, J. A.; Takanami, T. J. Am. Chem. Soc. 1998, 120, 4021.

44. Singh, V.; Porichu, M. Tetrahedron 1996, 52, 7087.

45. Miyashita, M.; Suzuki, T.; Hoshino, M.; Yoshikoshi, A. Tetrahedron 1997, 33, 12469.

46. Colvin, E. W.; Thom, I. G. Tetrahedron 1986, 42, 3137. 